\title{
Atomic-Scale Identification of Planar Defects in Cesium Lead Bromide Perovskite Nanocrystals
}

Arashdeep Singh Thind ${ }^{1}$, Guangfu Luo ${ }^{2}$, Jordan A. Hachtel ${ }^{3}$, Mariia Goriacheva ${ }^{4}$, Sung Beom Cho ${ }^{2}$, Albina Borisevich ${ }^{5}$, Juan Carlos Idrobo ${ }^{3}$, Yangchuan Xing ${ }^{4}$, and Rohan Mishra ${ }^{2,1}$

1. Institute of Materials Science \& Engineering, Washington University in St. Louis, St. Louis MO, USA

2. Department of Mechanical Engineering \& Materials Science, Washington University in St. Louis, St. Louis, MO, USA

3. Center for Nanophase Materials Sciences, Oak Ridge National Laboratory, Oak Ridge, TN, USA

4. Department of Chemical Engineering, University of Missouri, Columbia, MO, USA

5. Material Science and Technology Division, Oak Ridge National Laboratory, Oak Ridge, TN, USA

Lead-halide perovskites have emerged as a promising class of highly efficient and affordable semiconductors with applications in solar cells and optoelectronic devices [1]. Nanocrystals of inorganic lead-halide perovskites, such as $\mathrm{CsPBBr}_{3}$, display bright photoluminescence with a narrow emission bandwidth due to strong quantum confinement. Furthermore, the emission wavelength of these nanocrystals can be efficiently tuned over a wide spectral range either by changing their size or the anion composition ( $\mathrm{Cl}, \mathrm{Br}$ and I) [2]. One of the key features of these lead-halide perovskites that has been attributed to their success as high-performance semiconductors is their defect tolerance [3], which is an ability to retain the electronic structure of the pristine material even in the presence of a large concentration of defects. Their reported defect-tolerance, to a large extent, is based upon first-principles densityfunctional theory (DFT) calculations of the formation energy of point defects and their effect on the electronic structure. Unlike in conventional semiconductors, wherein point defects often lead to deeplevel defect states within the band gap that act as trap sites, point defects with low formation energy in lead-halide perovskites either lead to shallow defects levels or resonant levels within the valence or conduction bands [4]. In addition to the point defects, line- and planar-defects present in these perovskites may also have a large effect on their electronic structure. While there are theoretical reports on the electronic structure of hypothetical stoichiometric grain boundaries [5], direct observations of the atomic structure of dislocations, grain boundaries, twin boundaries and antiphase boundaries (APBs) are required so that accurate theoretical models could be built to evaluate their effect on the electronic structure.

In this work, we will present results showing the atomic structure and chemistry of line defects and planar defects in $\mathrm{CsPbBr}_{3}$ nanocrystals using aberration-corrected scanning transmission electron microscope (STEM) imaging and electron energy-loss spectroscopy (EELS). We have combined the atomic structure obtained from STEM-EELS with DFT calculations to elucidate the electronic structure of the perovskite nanocrystals in the presence of such defects.

We have used colloidal synthesis along with a novel fusion process to grow large nanocrystals of $\mathrm{CsPbBr}_{3}$ of size $>40 \mathrm{~nm}$ by merging smaller nanocrystals of size <10 $\mathrm{nm}$. Via STEM imaging, we observe dislocations and twinned domains in the smaller nanocrystals. In the larger nanocrystals, we observe an extensive network of APBs and low-angle grain boundaries that are formed during the fusion process. Figure 1a shows a high-angle annular dark field (HAADF) image of a non-stoichiometric $\sum 5$ grain boundary (Region-I) and an APB made up of an additional layer of CsBr with the rock salt structure (Region-II). We have used total energy DFT calculations to find the optimized structure of these defects, which are in excellent agreement with the STEM results, as shown in Figure 1b and 1c, respectively. Generally, in conventional semiconductors planar defects - such as grain boundaries and APBs - are 
detrimental to optoelectronic devices as they can introduce mid-gap states, which can act as nonradiative recombination centers. However, our DFT calculations reveal that neither the $\sum 5$ grain boundary nor the APB defects induce mid-gap states, as shown in Figure 1d, which lends support to the defect-tolerant nature of these perovskites. Moreover, we find that the APB defects introduce a potential barrier significant enough to cause quantum confinement of the electrons. Overall, our results provide insights into the defect structure and their effect on the properties of $\mathrm{CsPbBr}_{3}$ nanocrystals along with knowledge of chemical control over defects to achieve novel functionalities [6].

\section{References:}

[1] A. Kojima et al., J. Am. Chem. Soc. 131, 6050 (2009).

[2] L. Protesescu et al., Nano Lett. 15, 3692 (2015).

[3] W. J. Yin, T. Shi, and Y. Yan, Adv. Mater. 26, 4653 (2014).

[4] W.-J. Yin, T. Shi, and Y. Yan, Appl. Phys. Lett. 104, 063903 (2014).

[5] Y. Guo, Q. Wang, and W. A. Saidi, J. Phys. Chem. C 121, 1715 (2017).

[6] A.S.T. and R.M. acknowledge the support from National Science Foundation (NSF EAR -1726165).

This work was supported by a Ralph E. Powe Junior Faculty Enhancement Award from Oak Ridge Associated Universities to R.M. A portion of the microscopy research was performed as part of a user proposal at Oak Ridge National Laboratory's Center for Nanophase Materials Sciences (CNMS), which is a U.S. Department of Energy, Office of Science User Facility. This work used the computational resources of XSEDE, which is supported by NSF grant number ACI-1053575. A.B. was supoorted by the Division of Materials Science and Engineering, US DOE.
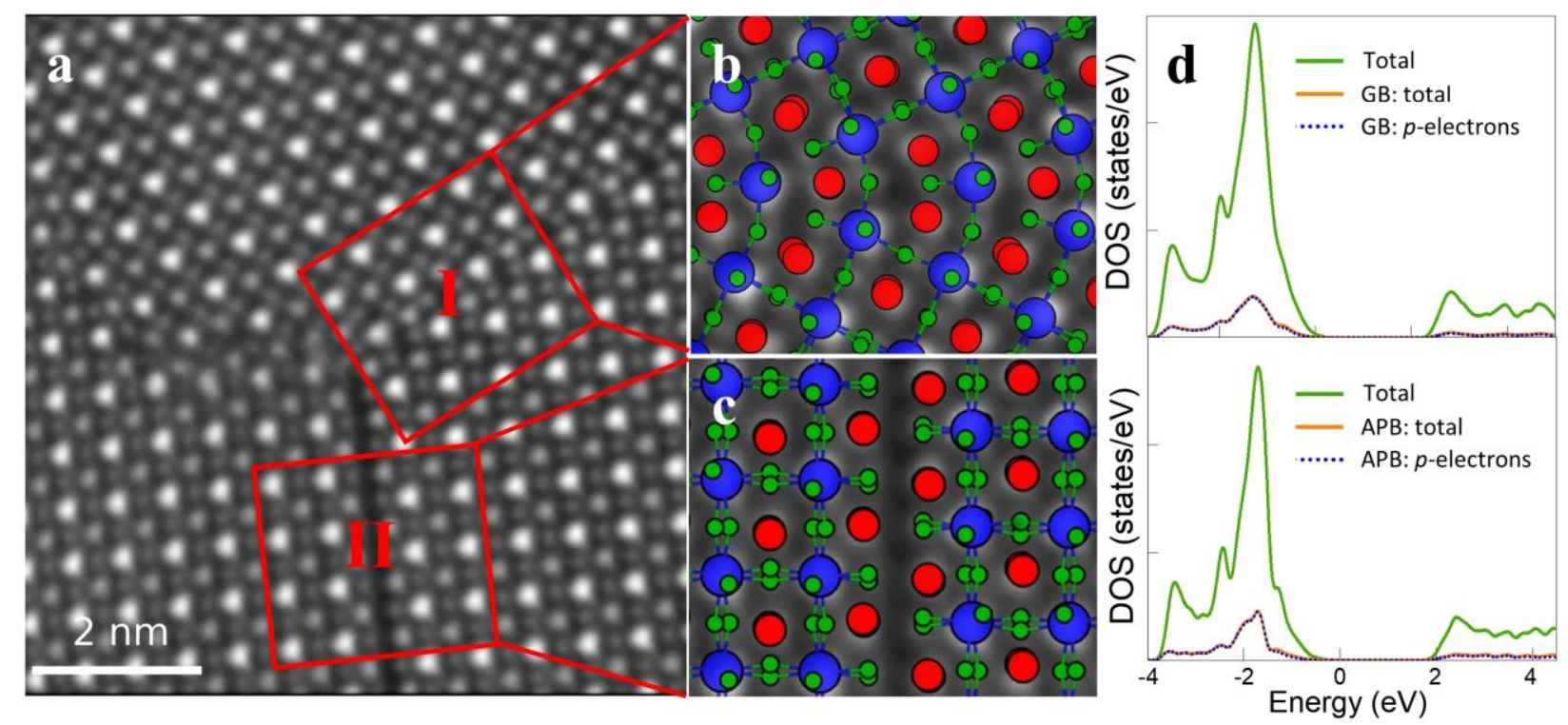

Figure 1. (a) HAADF image showing the $\Sigma 5$ grain boundary (Region-I) and APB (Region-II). DFT optimized crystal structure of (b) the $\Sigma 5$ grain boundary and (c) APB superimposed on respective parts of the image in (a). The blue, red and green atomic columns correspond to $\mathrm{Pb}, \mathrm{Cs}$ and $\mathrm{Br}$ respectively. (d) Density of states (DOS) plot for the $\Sigma 5$ grain boundary (top) and APB (bottom). 